\title{
IONIC MASS TRANSFER ON FIXED DISK AND CONICAL ELECTRODES UNDER STREAMING SOLUTIONS-I. THEORETICAL APPROACH*
}

\author{
S. L. Marchiano and A. J. Arvía \\ Instituto Superior de Investigaciones, Facultad de Quimica y Farmacia \\ and \\ Departamento de Ingenieria Quimica, Facultad de Ciencias Fisicomatematicas, \\ Universidad Nacional de La Plata, La Plata, Argentina
}

\begin{abstract}
A solution of the convective-diffusion differential equation for the case of disk and conical electrodes axially placed in laminar flow is attempted. The integration of the equations is done with an approximate streaming function which is valid for values of the function up to 0.3 within an error of one per cent.
\end{abstract}

The rate equation for a steady convective-diffusion process on the disk electrode is

$$
j=0.780 D_{i}\left(\frac{U}{\nu r}\right)^{1 / 2} \mathrm{Sc}^{1 / 3}\left(C_{i}^{0}-C_{i}{ }^{3}\right) \text {. }
$$

The type of solution is then extended to the case of conical electrodes with axial symmetry. A similar equation is thus obtained and the numerical coefficients for the average rate equation are in agreement with the one of the above equation, within 4 per cent, and are independent of the cone angle. The latter result agrees also with a previous equation deduced by analogy with the corresponding heat-transfer problem.

Résumé-Recherehe d'une solution de l'équation différentielle de diffusion-convection dans le cas d'électrodes d disque et conique axiales à un flux lamellaire. L'intégration des équations est tentée au moyen d'une fonction de courant approximative, valable avec une erreur d'un pour cent, pour des valeurs inférieures à 0,3 .

L'équation de débit pour un processus stationnaire de diffusion-convexion sur l'électrode à disque est:

$$
j=0,780 D_{i}\left(\frac{U}{v r}\right)^{1 / 2} \mathrm{Sc}^{1 / 3}\left(C_{i}{ }^{0}-C_{i}{ }^{2}\right) .
$$

Ce type de solution est ensuite étendu au cas d'électrodes coniques avec symétrique axiale. Une équation similaire est ainsi obtenue et les coefficients numériques intervenant dans l'équation donnant le débit moyen concordent à 4 pour cent près avec ceux impliqués avec l'équation au-dessus; ils sont indépendants de l'angle du cone. Ce dernier résultat est aussi en accord avec une équation préliminaire déduite par analogie avec le problème correspondant d'un transfert de chaleur.

Zusammenfassung-Es wurde versucht für den Fall von Scheiben- und Konus-Elektroden, welche sich achsial in einer laminaren Strömung befinden eine Lösung der Differentialgleichung des konvektiven Stofftransports zu gewinnen. Die Integration wurde mittels einer approximierten Strömungsfunktion durchgeführt, welche bis zu Werten der Funktion von 0,3 mit einem Fehler von weniger als 1 Prozent gilt.

Für den Fall des stationären Konvektions/Diffusionsvorganges an der Scheibenelektrode ergibt sich folgende Transportgleichung:

$$
j=0,780 D_{i}\left(\frac{U}{v r}\right)^{1 / 2} \mathrm{Sc}^{1 / 8}\left(C_{i}^{0}-C_{i}\right)^{2} \text {. }
$$

Die Lösungsmethode wird dann für den Fall der konischen Elektrode mit achsialer Symmetrie übernommen. Dabei wird eine analoge Lösung erhalten. Die numerischen Koeffizienten für die mittlere Stofftransportgeschwindigkeit stimmen mit denjenigen der obigen Gleichung innerhalb von 4 Prozent überein und sind anabhängig vom Konuswinkel. Dieses Ergebnis ist in Uebereinstimmung mit der früher unter Zuhilfenahme der Lösung für das analoge Wärmetransportproblem erhaltenen Gleichung.

* Manuscript received 25 July 1966. 


\section{INTRODUCTION}

THERE are various possibilities for increasing the rate of mass transfer in electrochemical reactions. One of the more detailed studies refers to the rotating disk electrode where the corresponding convective-diffusion differential equation has been satisfactorily solved ${ }^{1}$ on the basis of a known exact solution of the hydrodynamic equations. ${ }^{2.3}$ This electrode presents a uniformly accessible reaction surface, ${ }^{4}$ from the standpoint of diffusion. Recently a translating electrode ${ }^{5}$ was described offering the same advantages as the rotating disk electrode.

In the literature several attempts are mentioned where an increase of the rate of mass transfer was achieved by flowing the electrolyte solution while the working electrode was static. ${ }^{b-8}$ One of these refers to an arrangement of a conical electrode under a streaming solution. This electrode was used as a solid indicator electrode in hydrodynamic voltammetry. The interpretation given by the authors to the ionic mass-transfer rate assumed that under laminar flow and geometrical conditions similar to those prevailing on the electrode the solutions of the differential equations for heat transfer ${ }^{9}$ could be extended to ionic mass transfer.

The present study has a double purpose: first, to attempt the solution of the convective-diffusion differential equation for either fixed disk or conical electrodes in flowing solutions, to obtain an explicit function relating the current density with the variables which affect the rate of ionic mass transfer comprising the solution for fixed disk electrodes as a limiting case of the problem of conical electrodes; secondly, to verify experimentally the theoretical deductions. The former aspect is reported in this Part I and the latter in Parts II and III.

In order to solve the theoretical problem, the convective-diffusion differential equation of a fixed disk electrode with perpendicular flow is treated and then the solution of the problem is attempted for flow past a wedge. Finally, this second solution is extended to conical electrodes and compared and correlated with that for the disk-type fixed electrode.

\section{THE FIXED DISK ELECTRODE}

To deal with this problem we study the fixed disk electrode under a streaming solution impinging normally upon it with a model of disk in stagnation in threedimensional flow. ${ }^{10}$ We follow the mathematical procedure recently indicated for the translating electrode..$^{5}$ For the case of stagnation in three-dimensional flow, there is an exact solution of the hydrodynamical problem, which yields the radial and axial velocity distributions, $u$ and $w$, respectively, as a function of the following dimensionless variable,

$$
\xi=z \sqrt{\left(\frac{a}{v}\right)}
$$

where $z$ is a co-ordinate normal to the disk surface, $v$ is the kinematic viscosity of the fluid and $a$ is a constant given by

$$
U=a r
$$

$U$ is the potential flow and $r$ the radial co-ordinate. The axial velocity distribution function is

$$
w=-2 \sqrt{ }(a v) \phi(\xi)
$$


$\phi(\xi)$ is the distribution function in terms of $\xi$, given by (1), which is already known and tabulated for different values of $\sqrt{ }(2) \xi .10$

In the present case, the mass-transfer differential equation becomes

$$
w \frac{\partial C}{\partial z}=D_{i} \frac{\partial^{2} C}{\partial z^{2}},
$$

since the mass transfer occurs only in the $z$-direction towards the disk. The boundary conditions of (4) are

$$
\begin{array}{ll}
z=0, & C_{i}=C_{i}{ }^{\circ} ; \\
z=\alpha, & C_{i}=C_{i}^{\circ} .
\end{array}
$$

Taking into account the variable $\xi,(4)$ is converted into the total differential equation

$$
\frac{\mathrm{d}^{2} C_{i}}{\mathrm{~d} \xi^{2}}+2 \operatorname{Sc} \phi(\xi) \frac{\mathrm{d} C_{i}}{\mathrm{~d} \xi}=0,
$$

where $S c$ is the Schmidt number defined by the ratio $\nu / D_{i}, D_{i}$ is the diffusion coefficient of species $i$ and $C_{i}$ is its concentration. The boundary conditions in (6), in terms of the variable $\xi$, are

$$
\begin{array}{ll}
\xi=0, & C_{i}=C_{i}{ }^{\circ} ; \\
\xi=\infty, & C_{i}=C_{i}^{\circ} .
\end{array}
$$

The first integration of (6) gives

$$
\frac{\mathrm{d} C_{i}}{\mathrm{~d} \xi}=I_{1} \exp \left[-2 \mathrm{Sc} \int_{0}^{\xi} \phi(\xi) \mathrm{d} \xi\right],
$$

and with a second integration we obtain

$$
C_{i}=I_{1} \int_{0}^{\xi} \exp \left[-2 \mathrm{Sc} \int_{0}^{\xi} \phi(\xi) \mathrm{d} \xi\right] \mathrm{d} \xi+I_{2}
$$

The integration constants $I_{1}$ and $I_{2}$ are obtained from the boundary conditions. They are

and

$$
I_{1}=\frac{C_{i}^{\circ}-C_{i}{ }^{\circ}}{\int_{0}^{\infty} \exp \left[-2 \mathrm{Sc} \int_{0}^{\infty} \phi(\xi) \mathrm{d} \xi\right] \mathrm{d} \xi}
$$

$$
I_{2}=C_{i} \text {. }
$$

Considering (10) and (11) in (9) we have

$$
\frac{C_{i}-C_{i}}{C_{i}^{\circ}-C_{i}}=\frac{\int_{0}^{\xi} \exp \left[-2 \mathrm{Sc} \int_{0}^{\xi} \phi(\xi) \mathrm{d} \xi\right] \mathrm{d} \xi}{\int_{0}^{\infty} \exp \left[-2 \mathrm{Sc} \int_{0}^{\infty} \phi(\xi) \mathrm{d} \xi\right] \mathrm{d} \xi} .
$$


In order to solve the integrals of (12), we apply the numerical solution given for $\phi(\xi){ }^{10}$ The numerical data are approximated by

$$
\phi(\xi)=\alpha \xi^{2} .
$$

With a deviation less than 1 per cent for values of $\xi$ lower than $0.5, \alpha$ is a constant which is evaluated by plotting the tabulated results ${ }^{10}$ according to (13), just as for the case of stagnation in bi-dimensional fiow. ${ }^{5}$

From (12) and (13) we now obtain:

$$
\frac{C_{i}-C_{i}{ }^{s}}{C_{i}{ }^{\circ}-C_{i}{ }^{8}}=\frac{\int_{0}^{\xi} \exp \left[-2 \mathrm{Sc} \int_{0}^{\xi} \alpha \xi^{2} \mathrm{~d} \xi\right] \mathrm{d} \xi}{\int_{0}^{\infty} \exp \left[-2 \mathrm{Sc} \int_{0}^{\infty} \alpha \xi^{2} \mathrm{~d} \xi\right] \mathrm{d} \xi} .
$$

After performing the first integration, the integral of the denominator can be transformed into a gamma function if the variable $x$ is defined as

$$
x=\frac{2}{3} \operatorname{Sc} \alpha \xi^{3} .
$$

Then, the denominator of (14) becomes

$$
\int_{0}^{\infty} \exp \left[-\frac{2}{3} \mathrm{Sc} \alpha \xi^{3}\right] \mathrm{d} \xi=\left(\frac{3}{2 \alpha \mathrm{Sc}}\right)^{1 / 3} \frac{1}{3} \int_{0}^{\infty} \mathrm{e}^{-x} x^{-2 / 3} \mathrm{~d} x
$$

Equation (14) is therefore given by

$$
\frac{C_{i}-C_{i}^{s}}{C_{i}{ }^{\circ}-C_{i}{ }^{s}}=\left(\frac{2 \alpha \mathrm{Sc}}{3}\right)^{1 / 3} 1 \cdot 12 \int_{0}^{\xi} \exp \left[-2 \mathrm{Sc} \frac{\alpha}{3} \xi^{3}\right] \mathrm{d} \xi .
$$

Now we are ready to find an expression for the flux $j_{i}$, defined by Fick's law as

$$
j_{i}=D_{i}\left(\frac{\partial C_{i}}{\partial z}\right)_{z=0}=D_{i}\left(\frac{\partial C_{i}}{\partial \xi}\right) \cdot\left(\frac{\mathrm{d} \xi}{\mathrm{d} z}\right)_{\xi=0} .
$$

Actually we are interested in the maximum flux, $j_{\max }$, which is determined by the condition $C_{i}{ }^{s}=0$. By differentation of (17), and taking into account (18), we obtain

$$
j_{\max }=1 \cdot 12\left(\frac{2}{3} \alpha\right)^{1 / 3} D_{i} C_{i}\left(\frac{U}{\nu r}\right)^{1 / 2} \mathrm{Sc}^{1 / 3}
$$

For the case of stagnation in three dimensional flow, $\alpha$ is equal to 0.515 and by solving the numerical coefficients involved in (19) we get

$$
j_{\max }=0.780 D_{i} C_{i}\left(\frac{U}{v r}\right)^{1 / 2} \mathrm{Sc}^{1 / 3} .
$$

Therefore, under laminar flow conditions, we find that the maximum rate of mass transfer is directly proportional to the concentration of the diffusing species, to the square root of the fluid velocity and to the cube root of the Schmidt number. Relationships of the same form are normally found in mass-transfer phenomena under laminar flow. 


\section{CONICAL ELECTRODES UNDER LAMINAR \\ AXIAL STREAMING SOLUTIONS}

To approximate a solution to the mass-transfer differential equation applicable to conical electrodes we first consider a system comprising a flow past a wedge as indicated in Fig. 1. The solution of the hydrodynamical problem involved in such a system was

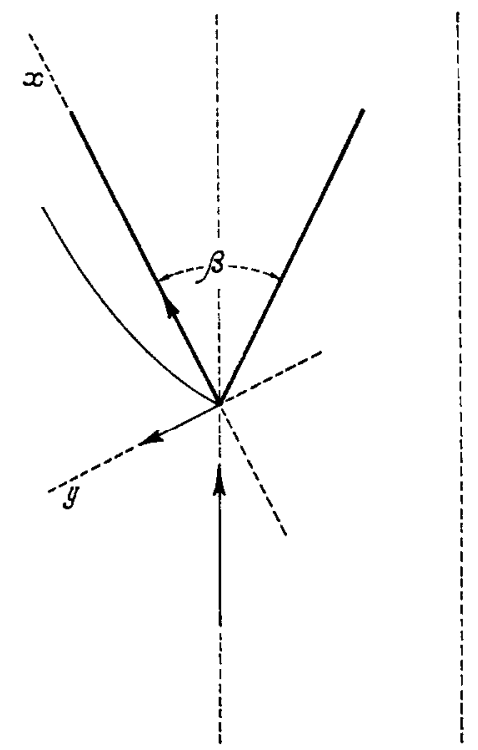

FIG. 1

attempted by Falkner and Skan, ${ }^{11}$ who obtained a dimensionless differential equation for this type of flux, which was later numerically solved. ${ }^{12}$ This approximation leads us to the following velocity distribution equations in the hydrodynamical boundary layer,

and

$$
u=U(x) f^{\prime}(\eta)=u_{1} x^{m} f^{\prime}(\eta)
$$

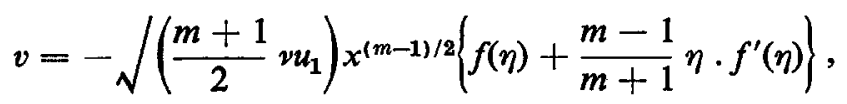

where $u$ and $v$ are the corresponding functions in the $x$ and $y$ directions respectively, $U(x)$ is the potential flux, $f(\eta)$ is the dimensionless streaming function and $f^{\prime}(\eta)$ is its first derivative, $\eta$ being a dimensionless variable given by

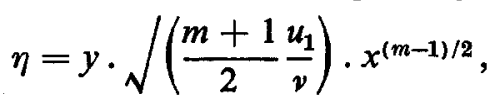

and $m$ being a number defined as

$$
m=\frac{\beta}{2-\beta},
$$

where $\beta$ is the angle indicated in Fig. 1 .

In this case the mass-transfer differential equation includes

$$
v \frac{\partial C}{\partial y}+u \frac{\partial C}{\partial x}=D_{i} \frac{\partial^{2} C}{\partial y^{2}},
$$

where the symbols have their already known meanings. 
To convert (25) into a total differential equation we follow the same procedure as described for the planar electrode, expressing it in terms of the dimensionless variable $\eta$, earlier given in the resolution of the hydrodynamical equations. So, taking into account (23), by substitution of $u$ and $v$ according to (21) and (22) respectively we obtain

$$
\begin{aligned}
& D_{i} \frac{m+1}{2} \frac{u_{1}}{\nu} x^{m-1} \frac{\mathrm{d}^{2} C}{\mathrm{~d} \eta^{2}}+\sqrt{\left(\frac{m+1}{2} u_{1} \nu\right) x^{(m-1) / 2}} /\left(\frac{m+1}{2} \frac{u_{1}}{\nu}\right) x^{(m-1) / 2} \\
\times & \left\{f(\eta)+\frac{m-1}{m+1} \eta f^{\prime}(\eta)\right\} \frac{\mathrm{d} C}{\mathrm{~d} \eta}-u_{1} x^{m} f^{\prime}(\eta) y \sqrt{\left(\frac{m+1}{2} \frac{u_{1}}{\nu}\right) \frac{m-1}{2} x^{(m-3) / 2} \frac{\mathrm{d} C}{\mathrm{~d} \eta}=0 .}
\end{aligned}
$$

After rearranging and simplification (26) becomes

$$
\frac{\mathrm{d}^{2} C}{\mathrm{~d} \eta^{2}}+\operatorname{Sc} f(\eta) \frac{\mathrm{d} C}{\mathrm{~d} \eta}=0
$$

This equation is analogous to (6), which was defined for the plane, and consequently, the analytical resolution of (27) at a constant angle yields an equation of the same form as (12).

An approximate solution of $(27)$ can be achieved as already indicated, assuming in this case a quadratic relationship between $f(\eta)$ and $\eta$, as seen in (13) for $\phi(\xi)$. The constant $\alpha^{\prime}$ can then be calculated from the numerical compilation of Hartree ${ }^{12}$ for $f^{\prime}(\eta)$ and $\eta$, after the former is conveniently integrated. In this way the $\alpha^{\prime}$ values corresponding to different values of $m$ in the range from 0 to 1 were calculated. They are assembled in Table 1 and appear as a function of $m$.

TABLE 1

\begin{tabular}{lcccc}
\hline$m$ & $\sqrt{\left(\frac{m+1}{2}\right)}$ & $\frac{m-1}{2}$ & $\alpha^{\prime}$ & $F$ \\
\hline 0 & 0.707 & -0.50 & 0.235 & 0.754 \\
0.1 & 0.742 & -0.45 & 0.285 & 0.808 \\
0.5 & 0.860 & -0.25 & 0.425 & 0.835 \\
1.0 & 1.000 & 0.00 & 0.515 & 0.807 \\
\hline
\end{tabular}

Following the procedure mentioned the following expression is obtained from (27) for the maximum local flux corresponding to a distance $x$ for the model previously described,

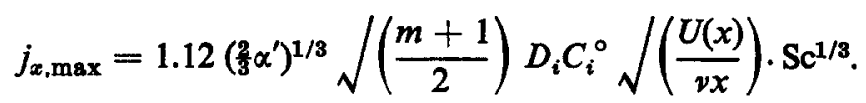

In order to extend it to a geometry comprising an axially symmetrical body and to evaluate an average flux, we apply to (25) the co-ordinate transformations of Manglen ${ }^{10}$ for this type of problem. The following transformation equations are required,

$$
\begin{gathered}
x=\frac{1}{L^{2}} \int_{0}^{x^{\prime}} r^{2}\left(x^{\prime}\right) \mathrm{d} x^{\prime}, \\
U(x)=U^{\prime}\left(x^{\prime}\right),
\end{gathered}
$$


where the dashes indicate the variables related to the axially symmetrical system. As deduced from Fig. $1, r\left(x^{\prime}\right)=x^{\prime} \sin \beta / 2$. Then, if the characteristic length, $L$, is defined as

after integration we have

$$
L=r=x^{\prime} \sin \beta / 2 \text {, }
$$

$$
x=x^{\prime} / 3 .
$$

By substitution of $x^{\prime}$ in (28) and taking into account that $U(x)=u_{1} x^{m}$, we get for the maximum average flux on the surface

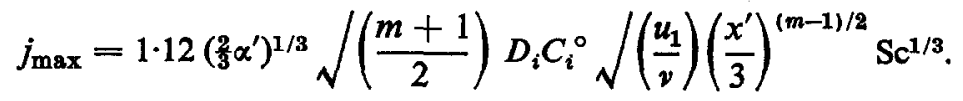

As $\alpha^{\prime}$ is a function of $m$, from (33), the factor $F$ comprising the terms which are a function of the conic angle is found,

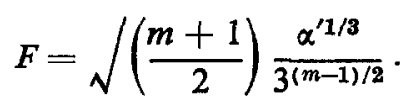

The values of $F$ calculated with values of $\alpha^{\prime}$ approximated according to (13) are given in Table 1 for values of $m$ from 0 to 1 . The figures are constant within 6 per cent, and suggest that the angle of the cone has no appreciable influence on the mass-transfer rate. Therefore, taking an average value of $F$, we can write for the maximum flux

$$
j_{\max }=0.80 D_{i} C_{i}^{\circ}\left(\frac{U^{\prime}\left(x^{\prime}\right)}{\nu x^{\prime}}\right) \mathrm{Sc}^{1 / 3}
$$

This equation has a numerical coefficient which coincides within 4 per cent with the one of (20).

\section{APPLICATION TO ELECTROCHEMICAL REACTIONS UNDER CONVECTIVE-DIFFUSION CONTROL}

The steady flux of matter of component $i$ involved in an electrochemical reaction, where migration of species $\mathrm{i}$ is negligible, is related to the current density, $i$, by the expression:

$$
i=n_{1} F D_{1}\left(\frac{\partial C_{1}}{\partial y}\right)_{y=0},
$$

where $n_{1}$ is the number of electrons entering the electrode process. Taking into account (17) and (18) in (36) we have

$$
i=0.80 n_{1} F D_{1}\left(\frac{U^{\prime}\left(x^{\prime}\right)}{v x^{\prime}}\right)^{1 / 2} \mathrm{Sc}^{1 / 3}\left(\frac{\mathrm{d} C}{\mathrm{~d} \eta}\right)_{\eta=0},
$$

and by solving $(\mathrm{d} C / \mathrm{d} \eta)_{\mathfrak{l}=0}$ in terms of the results given by differentiation of the concentration distribution equation derived from (27), we find

$$
i=0.80 n_{1} F D_{1}\left(\frac{U^{\prime}\left(x^{\prime}\right)}{\nu x^{\prime}}\right)^{1 / 2} \mathrm{Sc}^{1 / 3}\left(C_{1}^{\circ}-C_{1}{ }^{\circ}\right),
$$

and the maximum diffusional current density, $i_{\mathrm{diff}}$, becomes

$$
i_{\mathrm{diff}}=0.80 n_{1} F D_{1}\left(\frac{U^{\prime}\left(x^{\prime}\right)}{\nu x^{\prime}}\right)^{1 / 2} \mathrm{Sc}^{1 / 3} C_{1}^{\circ} .
$$


This equation bears the form already established for the rate of mass transfer under laminar flow at the rotating disk electrode, ${ }^{1}$ and for a flux along a plane electrode. ${ }^{13}$

As in other cases, the limiting current density increases linearly with the concentration of the reacting ion, and with the square root of the fluid velocity, and is inversely proportional to the square root of the electrode length. Consequently, with short length electrodes which still allow an appreciable current to be measured at a relatively low flow rate, it is possible to increase the current to such high values that the electrochemical process is no longer under convective-diffusion control. This may be of particular interest for the study of electrochemical reactions with intermediate kinetics.

Experimental tests of (39) are described in Parts II and III. In Part III, reference is also made to application of this type of analysis to intermediate kinetic processes.

Acknowledgement-Partial financial support for this work from the Consejo Nacional de Investigaciones Cientificas y Técnicas of Argentina and the fellowship granted to S. L. M. by the same institution are acknowledged.

\section{REFERENCES}

1. V. Levich, Physico-chemical Hydrodynamics. Prentice-Hall, Englewood Cliffs N.J. (1962).

2. TH. von KÁman, $Z$. angew. Math. Mech. 1, 233 (1921); N.A.C.A. Report TM 1052 (1946).

3. W. G. Cochran, Proc. Camb. phil. Soc. 30, 365 (1934).

4. D. A. Frank-Kamenetskin, Diffusion and Heat Exchange Chemical Kinetics. Princeton University Press (1955).

5. G. R. Bopp and D. M. Mason, Electrochem. Technol. 2, 129 (1964).

6. J. JoRDAN, R. A. JAVICK and W. E. RANZ, J. Am. chem. Soc. 80, 3846 (1958).

7. J. C. BAZÁn and A. J. ARVIA, Electrochim. Acta 9, 17 (1964).

8. J. C. BAZÁn and A. J. ARví, Electrochim. Acta 9, 667 (1964).

9. H. A. Jornson and M. W. Rubesin, Trans. Am. Soc. Mech. Engrs 71, 447 (1949).

10. H. SchlichtING, Boundary Layer Theory. McGraw-Hill, New York (1960).

11. V. M. FALKNER and S. W. SkAN, Phil. Mag. 12, 865 (1931).

12. D. R. HARTREe, Proc. Camb. phil. Soc. 33, 223 (1937).

13. G. WRANGLEN and O. NILsson, Electrochim. Acta 7, 111 (1962). 